\title{
Smoking Cessation in Patients With Cancer: Treatment Advances and the Oncologist's Role
}

\author{
Presented by Paul Cinciripini, $\mathrm{PhD}$
}

\begin{abstract}
The harms of smoking cigarettes are well-known, and the benefits of smoking cessation are well-established. Smoking cessation is especially important for patients with cancer, because smoking compromises the effects of cancer treatment and shortens survival. Interventions to achieve tobacco abstinence include pharmacotherapy and counseling, and these often must be repeated. Patients should be encouraged at every juncture to continue attempts to stop smoking.
\end{abstract}

J Natl Compr Canc Netw 2017;15(5.5):748-750

Helping patients with cancer (or any person) to quit smoking cigarettes is not easy. The effort often requires repeated attempts. Paul Cinciripini, PhD, Professor and Chair, Department of Behavioral Science, and Director, Tobacco Treatment Program, The University of Texas MD Anderson Cancer Center, updated listeners at the NCCN 22nd Annual Conference about current advances and how they have impacted the 2017 NCCN Clinical Practice Guidelines in Oncology (NCCN Guidelines) for Smoking Cessation.

"It is most important to consistently engage with your patients, whether they quit or not. Smoking cessation is not a single discrete event. It can take more than a year. The thing to remember is that smoking cessation is not 'a one and done,"' Dr. Cinciripini stated.

Dr. Cinciripini also discussed the broader picture of nicotine dependence in our society. Currently, 16.7\% of men and $13.6 \%$ of women in the United States are smokers. Drilling down into the data shows that socioeconomic and education factors are associated with

Presented by Paul Cinciripini, PhD, Department of Behavioral Science and Tobacco Treatment Program, The University of Texas MD Anderson Cancer Center, Houston, Texas.

Dr. Cinciripini has disclosed that he has served on the scientific advisory board of Pfizer Pharmaceuticals, conducted educational talks sponsored by Pfizer on smoking cessation (2006-2008), and has received grant support from Pfizer.

Correspondence: Paul Cinciripini, PhD, The University of Texas MD Anderson Cancer Center, Cancer Prevention Building, Room CPB3.3309, 1155 Pressler Street, Houston, TX 77030.

E-mail: pcinciri@mdanderson.org smoking. The rate of smoking is $26 \%$ for people below poverty level and $14 \%$ for those at or above poverty level. Rates of smoking show a large disparity related to education level; the rate of smoking is 3.6\% among individuals who hold a graduate degree and increases to $24.2 \%$ for those with an education level below a high school diploma.

Thirty-one percent of all cigarettes are smoked by adults with mental illness; $40 \%$ of men and $34 \%$ of women with mental illness smoke, Dr. Cinciripini noted.

"Smoking disproportionately occurs among people with the fewest resources," he said. "There is a gradient across the age span where high and moderate levels of psychological distress are associated with higher levels of smoking," he added.

However, once people stop smoking, levels of depression, anxiety, and stress are reduced, and mood and quality of life are improved. "The effect sizes of stopping smoking on these parameters are equal to or larger than those of antidepressant treatment," Dr. Cinciripini continued.

Further, more than 480,000 deaths each year are attributable to smoking. Approximately $30 \%$ of lung cancers are smoking-related, as are $8 \%$ of other cancers.

\section{Smoking and Cancer}

Smoking has been linked to $>15$ types of cancer. Smoking can compromise the effects of cancer treatment and shorten survival. Patients who continue to smoke have an increased risk of recurrence and poor 
quality of life. For example, smoking in the perioperative period increases the risk of pulmonary embolism and poor wound healing. Smoking diminishes the efficacy of chemotherapy, impairs immune function, and increases the risk of infection.

Net benefits of quitting smoking include longer survival, improved chances of successful cancer treatment, fewer and less serious adverse events (AEs), increased energy, and better quality of life. A recent study found that smoking cessation after stereotactic body radiation therapy improved overall survival in patients with nonsmall cell lung cancer (Figure 1). At 2 years after treatment, $78 \%$ of those who stopped smoking were alive versus $69 \%$ of those who continued to smoke $(P=.014){ }^{1}$

"I think you will see more and more evidence like this as we integrate smoking cessation into our cancer centers," Dr. Cinciripini predicted. The need for intervention is great in patients with cancer. Dr. Cinciripini said that $68 \%$ of smokers want to quit, $55.4 \%$ report a serious attempt to quit within the past 22 months, and the annual rate of cessation is $7.4 \%$.

"It may take multiple attempts to quit smoking," Dr. Cinciripini noted. "Nicotine dependence is a chronic relapsing disorder. This means we have to continue to provide the opportunity for treatment. Negative affect predicts both prevalence and relapse rates. If you are not addressing the individual's mood, you will be missing an important component of nicotine addiction."

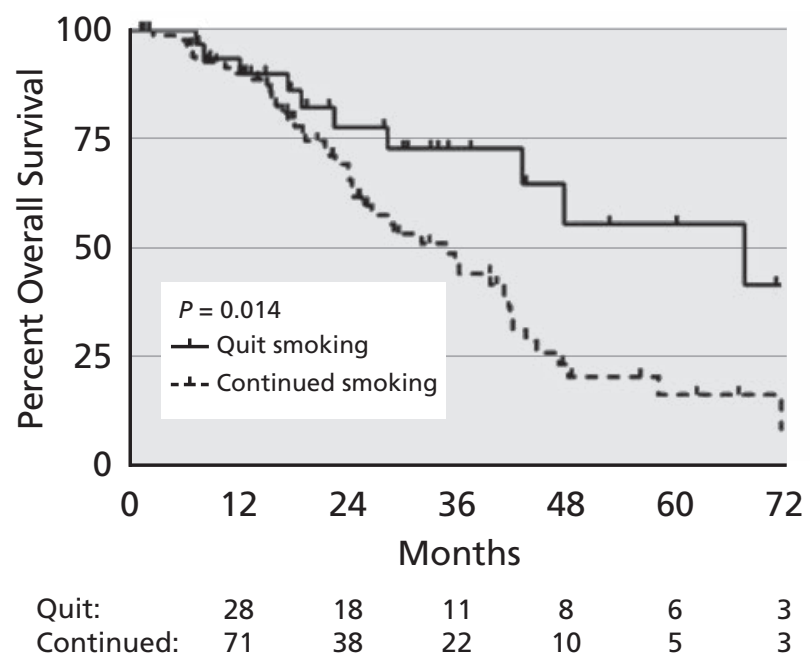

Figure 1. Smoking cessation after stereotactic body radiation therapy for non-small cell lung cancer.

From Roche MC, Rehman S, DeWees TA, et al. It's never too late: smoking cessation after stereotactic body radiation therapy for nonsmall cell lung carcinoma improves overall survival. Pract Radiat Oncol 2016;6:12,18. Reprinted with permission from Elsevier @2016.

\section{Evidence-Based Treatment Recommendations}

Both counseling and medications should be provided to all patients who smoke. The combination of these interventions is more effective than less intensive efforts.

First-line pharmacotherapies include nicotine replacement therapies (NRTs; eg, gum, patch, lozenge, and nasal spray). Other first-line choices are bupropion-SR and varenicline. Second-line choices are nortriptyline and clonidine. However, nortriptyline is problematic and the drug is cardiotoxic at higher doses, Dr. Cinciripini reminded listeners. "I don't recommend clonidine unless trials of other medications fail," he added.

Dr. Cinciripini's experience is that combinations of the nicotine patch with NRT lozenge or gum or varenicline represent the best approaches.

A meta-analysis suggested that greater improvement in achieving smoking cessation was associated with varenicline compared with other monotherapies. Combination NRT was the second-best approach. ${ }^{2}$ Dr. Cinciripini suggests using the NRT patch and lozenge or gum as first line in the oncologic setting if the practitioner is unfamiliar with varenicline or has some concern about the potential for neuropsychiatric side effects reported in postmarketing reports (eg, suicidal ideation, anxiety, depression).

However, the risk of such effects were directly examined in the largest randomized clinical trial for smoking cessation performed to date (EAGLES). ${ }^{3}$ The study enrolled approximately 8,000 patients: approximately 4,000 without and 4,000 with past or current psychiatric disorders, including those on psychotropic medication. In this study, varenicline outperformed NRT (patch), bupropion, and placebo in achieving abstinence at the end of treatment and at 6 months. Importantly, although the risk of neuropsychiatric events was generally higher among smokers in the psychiatric versus nonpsychiatric cohort, no significant differences were seen across treatments in neuropsychiatric events, including those rated as severe. ${ }^{3}$

"This study was instrumental (along with several previous observational studies) in the decision by the FDA to remove the black box warning associated with psychiatric AEs for varenicline." Nevertheless, Dr. Cinciripini noted that it is still important to ask about psychiatric history and symptoms 
when considering any of these medications, and to consider using varenicline when patients are stable (no change in medication or symptoms in past 3 months). Clinicians should follow guidelines of good clinical practice by following up with these patients a few weeks after starting medication to assess their progress and tolerance. Nausea and other gastrointestinal symptoms are the most common AEs associated with varenicline.

\section{Combination Therapies}

Studies have evaluated the combination of varenicline and bupropion. In one study of 506 adult cigarette smokers, the combination appeared to be more effective than varenicline plus placebo at week 12 , but no difference was seen between strategies at week $52 .{ }^{4}$

Dr. Cinciripini said that a separate study suggested that the combination may be more effective in men and in heavily dependent smokers. However, he believes that more evidence is needed before recommending the combination of varenicline and bupropion be used as a first-line option for all smokers, with the exception of a possible benefit in heavily dependent male smokers.

A separate study of 446 smokers found that varenicline plus NRT was more effective than varenicline alone in achieving tobacco abstinence at 12 weeks (end of treatment) and at 6 months. ${ }^{5}$ More studies of the combination of varenicline and NRT are needed, he said.

\section{When to Start Treatment?}

Although most people use the NRT patch beginning on their quit date, recent research suggests that using the patch before quitting and undergoing counseling at that time can achieve a better outcome.

"The rationale is that NRT can be used as a motivational tool. It cuts down nicotine cravings and teaches smokers they can go long periods of time without smoking," Dr. Cinciripini stated.
Dr. Cinciripini discussed his experience with 4,000 patients at The University of Texas MD Anderson Cancer Center. ${ }^{6}$ This experience revealed no major differences in the efficacy of smoking cessation intervention between people with or without cancer and between those who had smokingrelated cancers and those who did not, when using individually tailored approaches to counseling and pharmacotherapy.

\section{NCCN Guidelines for Smoking Cessation}

Dr. Cinciripini discussed the steps outlined in the NCCN Guidelines: first determine whether the patient is a current smoker and recent quitter (smoked within the last 30 days). Assess past quit attempts and attempts at treatment and ascertain if the person is ready to quit.

If the patient indicates a readiness to quit in the next 4 weeks, establish a treatment plan. If not, assess the barriers and concerns of the patient. "Here's where smoking reduction comes in," Dr. Cinciripini advised. "Consider smoking reduction with a goal of setting a quit date in the near future."

Choices of therapy are combination NRT and behavioral therapy for 12 weeks, or varenicline plus behavioral therapy for 12 weeks. If the person is smoke-free at that point, provide motivational strategies for continued abstinence and extend duration of pharmacotherapy if needed. Continue to reassess smoking status at 6 and 12 months after therapy.

If the patient has relapsed or is still smoking, continue the attempts at pharmacotherapy and counseling and consider intensifying behavioral therapy. Follow-up is important for patients to maintain high levels of motivation.

"At each juncture point, for a quitter or relapse prevention or switching to another therapy, continue to engage with your patient," he advised.

\section{References}

1. Roach MC, Rehman S, DeWees TA, et al. It's never too late: smoking cessation after stereotactic body radiation for non-small cell lung carcinoma improves overall survival. Pract Radiat Oncol 2016;6:12-18.

2. Mills EJ, Wu P, Lockhart I, et al. Comparisons of high-dose and combination nicotine replacement therapy, varenicline, and bupropion for smoking cessation: a systematic review and multiple treatment metaanalysis. Ann Med 2012;44:588-597.

3. Anthenelli RM, Benowitz NL, West R, et al. Neuropsychiatric safety of varenicline, bupropion, and nicotine patch in smokers with and without

psychiatric disorders (EAGLES): a double-blind, randomized, placebo controlled trial. Lancet 2016;387:2507-2520.

4. Ebert JO, Hatsukami DK, Croghan IT, et al. Combination varenicline and bupropion SR for tobacco dependence treatment in cigarette smokers: a randomized trial. JAMA 2014;311:155-163.

5. Koegelenberg CF, Noor F, Bateman ED, et al. Efficacy of varenicline combined with nicotine replacement therapy vs varenicline alone for smoking cessation: a randomized clinical trial. JAMA 2014;312:155-161.

6. Karam-Hage M, Oughli HA, Rabius V, et al. Tobacco cessation treatment pathways for patients with cancer: 10 years in the making. J Natl Compr Canc Netw 2016;14:1469-1477. 